\title{
Exercício físico como fator de prevenção aos processos inflamatórios decorrentes do envelhecimento
}

\author{
Ciro José Brito ${ }^{1,4}$ \\ Ana Carolina Pinheiro Volp ${ }^{2}$ \\ Otávio de Toledo Nóbrega ${ }^{3}$ \\ Fernando Lopes e Silva Júnior ${ }^{4}$ \\ Edmar Lacerda Mendes ${ }^{5}$ \\ Aendria Fernanda Castro Martins Roas ${ }^{6}$ \\ Jonatas de França Barros ${ }^{7}$ \\ Cláudio Córdova ${ }^{4}$
}

${ }^{1}$ Departamento de Educação Física, Núcleo de Pesquisa de em aptidão física de Sergipe, Universidade Federal de Sergipe, SE, Brasil

${ }^{2}$ Escola de Nutrição, Departamento de Nutrição Clínica e Social, Universidade Federal de Ouro Preto, MG, Brasil

${ }^{3}$ Programa de Pós-Graduação em Ciências Médicas, Faculdade de Medicina,

Universidade de Brasília, Brasília, DF, Brasil

${ }^{4}$ Programa de Pós-graduação Stricto Senso em Educação Física, Grupo de estudos em

Imunogenética e Exercício Físico, Universidade Católica de Brasília, DF, Brasil

${ }^{5}$ Programa de Pós-Graduação em Educação Física, Departamento de Ciências do Esporte, Laboratório Imunologia e Metabologia do Exercício e do Esporte, Universidade Federal do Triângulo Mineiro, Uberaba, MG, Brasil

${ }^{6}$ Centro Universitário Metodista de Minas Izabela Hendrix e Departamento de Programas Esportivos - Minas Tênis Clube, Belo Horizonte, MG, Brasil

7 Departamento de Educação Física/CCS, Programa de Pós-Graduação em Educação Física/UFRN. Programa de Doutorado em Ciências da Saúde/FS/UnB. Laboratório de Movimento Humano. Universidade Federal do Rio Grande do Norte, Natal, RN, Brasil

\begin{abstract}
Resumo: Ao longo do processo de envelhecimento observa-se complexa remodelagem do sistema imunitário. Estas alterações estão associadas ao desenvolvimento de patologias responsáveis por grande parte da mortalidade em população idosa. Recentemente, a prática regular de atividades físicas tem sido proposta como intervenção não-medicamentosa com amplos benefícios sobre a regulação de processos decorrentes da imunossenescênia. Neste sentido, o presente trabalho revisou e discutiu estudos que abordam a ação de mediadores pró-inflamatórios crônicos e possíveis ações do exercício físico como agente antiinflamatório. Baseado nos resultados de estudos na literatura sugere-se que, em conjunto, a interleucina-6 (IL-6) e o fator de necrose tumoral- $\alpha$ (TNF- $\alpha$ ) são as principais citocinas associadas à aterosclerose, sarcopenia e déficits cognitivos. Embora os mecanismos não sejam totalmente elucidados, 0 exercício reduz a atividade de citocinas pró-inflamatórias e aumenta a liberação de substancias antiinflamatórias.
\end{abstract}

Palavras-chave: Imunologia. Citocinas. Envelhecimento. Predisposição genética para doença. Exercício.

\section{Physical exercise as a preventive procedures inflammation of aging}

\begin{abstract}
During the aging is observed complex remodeling of immune system. These changes are associated with the development of diseases responsible for much of the mortality in the elderly. Recently, the regular practice of exercise has been proposed as an intervention non-medication with broad benefits on regulation processes arising from Immunosenescence. In sense, this paper reviewed and discussed studies addressing the action of pro-inflammatory mediator's chronic and possible actions of physical exercise as anti-inflammatory agent. Based on the results of studies in the literature suggest that, together, interleukin-6 (IL-6) and tumor necrosis factor- $\alpha$ (TNF- $\alpha$ ) are the main cytokines associated with atherosclerosis, sarcopenia and cognitive deficits. Though mechanisms are not fully elucidated, the Exercise reduces the activity of pro-inflammatory and increases the release of anti-inflammatory substances.
\end{abstract}

Key words: Immunology. Cytokines. Aging. Genetic predisposition to disease. Exercise. 


\section{Introdução}

Nos últimos anos, observa-se a transição de um modelo caracterizado por altas taxas de natalidade e mortalidade, em que a fecundidade é cada vez menor e a expectativa de vida, maior. No cerne dessa transição, verifica-se o aumento progressivo da proporção de idosos e a redução relativa de jovens. Nesta perspectiva, é imperativo que se identifique fatores de riscos e que se estabeleçam terapias efetivas para a prevenção/recuperação de patologias inerentes ao processo do envelhecer (NICKLAS; BRINKLEY, 2009).

Neste contexto, torna-se importante o desenvolvimento de tecnologias, principalmente na área da saúde e qualidade de vida para o atendimento desta população, pois, a elevada atividade inflamatória que acompanha 0 envelhecimento é parte característica de processos patológicos crônicos que representam a maior parte das causas de mortalidade em população idosa. O termo inflammaging é utilizado para descrever respostas orgânicas complexas a vários estímulos inflamatórios mediados por elevados níveis de citocinas pró-inflamatórias (GIUNTA, 2006; KRABBE et al., 2004).

$\begin{gathered}\text { Sugere-se que inflammaging seja } \\ \text { desencadeado mediante desorganização }\end{gathered}$
funcional crônica da primeira linha de defesa
orgânica, ou seja, do sistema imunitário inato que
opera com a função de detectar amplo espectro
de lesões e que durante a imunossenescência
induz exacerbada resposta inflamatória por
modificação no perfil de citocinas pró-
inflamatórias (FRANCESCHI et al., 2000;
CANDORE et al., 2006). Até o momento, não
existem tratamentos definitivos para a redução
desta condição (NICKLAS; BRINKLEY, 2009).
Alternativamente, a administração de
medicamentos antiinflamatórios é utilizada; porém
efeitos adversos limitam a aplicação clínica
indiscriminada para o tratamento de processos
inflamatórios persistentes.

Por outro lado, a prática regular de exercício tem sido apontada como intervenção nãomedicamentosa com amplos benefícios sobre sistemas fisiológicos que exibem deterioração funcional e estrutural com 0 curso do envelhecimento (ACSM, 2009). Entretanto, levanta-se a hipótese que sessões agudas de exercício podem induzir respostas inflamatórias que muito se assemelham àquelas decorrentes de processos infecciosos (PETERSEN; PERDENSEN, 2005). Neste sentido, persistem controvérsias a cerca dos potenciais benefícios do exercício como regulador da resposta inflamatória relacionada a doenças crônicodegenerativas e a imunossenescência (NICKLAS; BRINKLEY, 2009; FISCHER, 2006; KRABBE et al., 2004; NICKLAS, et al., 2004; FEBBRAIO; PEDERSEN, 2002). Com base em resultados de estudos experimentais e observacionais, o presente trabalho teve por objetivo revisar temas que abordam processos inflamatórios com o envelhecimento e possíveis interações do exercício como agente antiinflamatório.

\section{Revisão de Literatura}

O referencial teórico do presente trabalho foi escrito através de revisão bibliográfica sistemática com as palavras "exercise", "aging and exercise", "exercise and inflammation", "aging and inflammation mediators", "epidemiology, exercise and aging", nas seguintes bases de dados em saúde: MEDLINE (base de dados de literatura internacional, produzida pela US National Library of Medicine - NLM), LILACS (Literatura LatinoAmericana y del Caribe en Ciencias de la Salud), SciELO (Scientific Electronic Library Online), Periódicos da Capes, além de sites da Internet e livros da área.

\section{Aspectos imunitários e genéticos do organismo idoso}

Uma breve revisão da literatura científica mostrará vários estudos relacionando alterações das funções imunológicas e 0 processo de envelhecimento. FRANCESCHI et al. (2000) descrevem a imunossenescência como uma complexa remodelagem onde enquanto alguns parâmetros imunológicos diminuem com idade, outros aumentam ou permanecem inalterados. Assim, o fenômeno de imunossenescência compreende uma série de modificações, das quais se destaca sobremaneira 0 aumento da atividade inflamatória basal, definido como inflammaging. Em outras palavras, o termo inflammaging (inflammation + aging) vem sendo utilizado para designar uma sutil, porém sustentada regulação positiva da resposta inflamatória de etiologia idiopática que contribui para o surgimento das doenças inflamatórias (FRANCESCHI et al., 2000). De fato, as respostas inflamatórias estão envolvidas na patogênese de doenças relacionadas com a idade, uma vez que doença de Alzheimer, aterosclerose, diabetes e alguns cânceres apresentam importante componente inflamatório em suas respectivas fisiopatologias (FRANCESCHI et al., 2000).

Uma variedade de relatos tem demonstrado alterações em diversos componentes do sistema imunitário do idoso no que se refere à contagem global e diferencial de linfócitos $T$ e $B$ virgens, 
efetores e de memória, ao número e atividade de células Natural Killer (SANSONI et al., 1993), na freqüência de auto anticorpos órgão-específicos e não específicos (FRANCESCHI et al.,1995; MARRIOTTI et al., 1995), nos componentes e atividade do complemento (BELLAVIA et al., 1999), no número e função das células progenitores (células CD34+) e na produção de citocinas (IL-1, IL-3, IL-6,IL-7, IL8, IL-10, TNF- $\alpha$ e IFN-y) (BONAFÈ et al., 2001).

No que se referem aos fatores humorais considerados marcadores de inflamação aguda, tais como a Proteína C-Reativa (PCR), pode-se dizer que possuem forte valor preditivo para doenças cardiovasculares assim como isquemias, infartos e doenças coronarianas, sendo sua utilização disseminada na prática médica (HARRIS et al, 1999). Neste sentido, citocinas vem mostrando igual aplicabilidade no prognóstico de doenças associadas à idade. Em regra, indivíduos que apresentam dosagens elevadas de citocinas pró-inflamatórias têm demonstrado associação com doenças relacionadas ao envelhecimento, tais como Alzheimer (BHOJAK et al., 2000) e osteoporose (HOBSON; RALSTON, 2001). Como exemplo desta associação, dosagens elevadas de IL-6 têm sido associadas com maior prevalência de morbidades entre idosos, sendo assim considerado por diferentes autores como fator predisponente a incapacidade funcional $\mathrm{e}$ mortalidade comparável a PCR (HARRIS et al., 1999). Assim, citocinas despontam como fatores implicados com o processo de doenças crônicas degenerativas, uma vez que possui valor preditivo potencial para longevidade saudável.

Desta forma, a literatura científica corrobora a noção de que os diversos distúrbios degenerativos associados ao envelhecimento apresentam um forte componente inflamatório em sua fisiopatologia. Ademais, pesquisas vêm demonstrando que o fenômeno de inflammaging e as diferentes trajetórias clínicas envolvendo inflamação possuem um forte componente genético. Determinados polimorfismos de região promotora e de fase aberta de leitura tem sido insistentemente implicados com níveis séricos de mediadores inflamatórios. Por exemplo, um polimorfismo de repetição de dinucleotídeos CA no primeiro íntron do gene para IFN-y vem sendo associado com maior produção da referida citocina (PRAVICA, 1999). Ademais, outro polimorfismo para IFN-y foi apontado como provável marcador para inflammaging em mulheres (LIO, 2002). Mas o principal polimorfismo de citocina que vem sendo associado com doenças agudas e crônicas no envelhecimento consiste naquele polimorfismo de base única (SNP) na posição -174 do gene da IL6. Além dele, existem outros pontos singulares de polimorfismo na região promotora da IL-6, a saber: as variações $-597 \mathrm{G} / \mathrm{A}$ e $-572 \mathrm{G} / \mathrm{C}$. No entanto, essas variações encontram-se em desequilíbrio de ligação com o polimorfismo -174 C/G, de modo a se convencionar o estudo deste último (FISHMAN et al., 1998; GEORGES et al., 2001; TERRY et al., 2000). Acredita-se que essa mudança de bases $\mathrm{G} \rightarrow \mathrm{C}$ na posição -174 afeta $a$ transcrição do gene, podendo alterar os níveis plasmáticos da IL-6 (CHAVEZ et al., 2002), podendo ter significado relevante na suscetibilidade a determinadas patologias. Com relação à doença de Alzheimer, existe importante relação entre a presença do alelo $C$ com 0 aumento do risco de desenvolver a patologia (LICASTRO et al., 2003). E ainda, o polimorfismo $-174 \mathrm{C} / \mathrm{G}$ pode estar relacionado com o aumento do risco das doenças cardiovasculares (CVD) em idosos (JENNY et al., 2002). Neste caso, o alelo $C$ parece desempenhar um importante papel, principalmente no desenvolvimento da aterosclerose. No caso da patologia da osteoporose, tem-se que o genótipo CC está associado com baixa absorção e menor diminuição da massa óssea.

O entendimento dos mecanismos imunogenéticos contribuirá para o monitoramento do fenômeno de inflammaging na população brasileira, com vistas ao desenvolvimento de estratégias terapêuticas voltadas à promoção da qualidade de vida e diminuição da morbimortalidade relacionada a IL- 6 e TNF- $\alpha$ em idosos. No entanto, mesmo que se torne possível utilizar tais citocinas como marcadores de prognóstico de envelhecimento, precisar-se-à tornar a dosagem das mesmas algo acessível sob o ponto de vista econômico, uma vez que a tecnologia comercial ora disponível é dispendiosa, o que dificulta que tais análises sejam parte integrante da prática médica.

\section{Efeitos de mediadores inflamatórios em doenças crônicas relacionadas à imunossenescência.}

Doenças crônicas representam a principal causa de morte no mundo, lideradas por doenças cardiovasculares (17 milhões de óbitos/ano), câncer (7 milhões de óbitos/ano), doenças do trato respiratório (4 milhões de óbitos/ano) e diabetes mellitus (cerca de 1 milhão de óbitos/ano) (PEDERSEN; FISCHER, 2007). A forte associação entre os níveis de mediadores inflamatórios circulantes e o risco de mortalidade (PEDERSEN; FISCHER, 2007), independente de morbidades pré-existentes, sugere-se que 
citocinas desencadeiam/potencializam processos patológicos crônicos ou atuam como marcadores sensíveis de distúrbios subclínicos em idosos. Em linha com esta hipótese, observações epidemiológicas sugerem que doenças como aterosclerose, sarcopenia e declínio cognitivo compartilham de alguns fatores etiológicos no qual mediadores inflamatórios fornecem a possível base comum (KRABBE et al., 2004).

\section{Aterosclerose}

A aterosclerose consiste na formação de placas ateroscleróticas ou ateromas ao longo do leito vascular e envolvem acúmulo de lipídeos, migração, proliferação, morte celular e fibrose da parede arterial (SKOOG et al., 2002). Esta patologia é resultado de respostas inflamatórias a lesões vasculares e disfunção endotelial por combinações de fatores de risco (KRABBE et al., 2004). Células endoteliais ocupam posição estratégica na defesa e inflamação do hospedeiro, produzindo e reagindo a diversas citocinas. Neste sentido, níveis plasmáticos de TNF- $\alpha$ são correlacionados com dislipidemias, hipertensão, resistência insulínica em homens de meia idade (SKOOG et al., 2002) e elevada prevalência de DCV em idosos octogenários (BRUUNSGAARD et al., 2000). Estudos epidemiológicos também sugerem níveis circulantes de IL-6 como marcadores subclínicos de DCV, preditores de mortalidade e risco de mortalidade em pacientes com história de DCV (JENNY et al., 2002; HARRIS et al., 1999). Ademais, TNF- $\alpha$ e IL-6 afetam sistemas de coagulação, metabolismo de lipídeos (KRABBE et al., 2004; BRUUNSGAARD; PEDERSEN, 2003) e induzem a produção da Proteína C-reativa (PCR) que, por sua vez, tem se revelado como forte e consistente preditor de doenças coronarianas (PEPYS; HIRSCHFIELD, 2003).

Um crescente corpo de evidencias tem sugerido que, a função patogênica do mediador inflamatório IL-6 na DCV é mais provável de ser observada em portadores de polimorfismo promotor $-174 \quad \mathrm{G} / \mathrm{C}$ do gene IL-6 (BRUUNSGAARD et al., 2003; JENNY et al., 2002; HUMPHRIES et al., 2001). Mediadores inflamatórios podem induzir diversos fatores de risco nas DCV e contribuir com o desenvolvimento da síndrome metabólica: o TNFa promove resistência insulínica e disfunção endotelial incluindo prejuízos em mecanismos de relaxamento e aumento na expressão de moléculas de adesão (KRABBE et al., 2004).

Por outro lado, a prática de exercício físico tem sido associada com benefícios sobre o sistema cardiovascular (LEUNG et al. 2008). Por exemplo, o estudo populacional de Zhang et al. (2010) evidenciou que níveis mais elevados da adipocina resistina e incidência de isquemia cardíaca foram observados naqueles com menor aptidão física. Quanto ao tipo de exercício, MIERS (2003) sugere que 0 exercício aeróbio contribui positivamente para a redução da atividade aterogênica. Em contrapartida, GAVIN et al. (2010) revela que somente adaptações induzidas pelo exercício resistido reduzem a atividade aterogênica em diabéticos do tipo II. Em recente revisão, Brandt e Pedersen (2010) evidenciaram que o exercício físico é fundamental no controle da aterosclerose, pois a prática de exercícios apresenta como efeito crônico a redução na expressão de citocinas pró-inflamatórias como o TNF- $\alpha$, ademais a musculatura esquelética quando estimulada libera miocinas antiinflamatórias como a IL-6 e IL-15.

Em conjunto, estes resultados sugerem que o incremento dos níveis sistêmicos de mediadores pró-inflamatórios circulantes em população idosa constitui fator comum entre o estilo de vida, fatores de risco para DCV e alterações fisiológicas durante a imunossenescência.

\section{Síndrome de fragilidade e sarcopenia}

A síndrome de fragilidade é definida como um declínio gradual de massa corporal, força muscular, reduzida disposição física, perda de autonomia, hospitalização e mortalidade. A sarcopenia destaca-se como parte central dessa síndrome (ROTH, et al., 2006; KRABBE et al., 2004). Esse declínio tem início por volta da terceira década da vida, tornando-se mais significativo após os 65 anos de idade. Assim, aos 80 anos, o indivíduo apresenta redução média de $30 \%$ de massa muscular quando comparado a um adulto jovem (BAUMGARTNER et al., 1995; EVANS, 1995). Embora os mecanismos não sejam claros, a etiologia da sarcopenia parece ser multifatorial (ROTH et al., 2000; $\underline{\mathrm{ROTH}}$ et al., 2006; ROUBENOFF; HUGHES, 2000) e refletindo desequilíbrio metabólico causado por elevada produção de citocinas catabólicas e reduzida produção/disponibilidade de hormônios anabólicos (HAMERMAN, 1999).

Neste contexto, associações entre mediadores pró-inflamatórios, sarcopenia e perda na capacidade funcional sugerem que níveis circulantes de citocinas sejam importantes marcadores de subjacentes processos patológicos (KRABBE et al., 2004). Por exemplo, resultados de estudos com cultura de células musculares sugerem que o TNF- $\alpha$ contribui diretamente com a sarcopenia, uma vez que esta citocina interrompe processos de diferenciação 
celular, promove o catabolismo e a apoptose de células musculares (ROUBENOFF, 2003). Outro fator possível fator associado à sarcopenia é a redução da sinalização Notch (sinalização justácrina advinda do contato físico entre células adjacente) nas células musculares ao longo do processo de envelhecimento ( $\underline{\mathrm{CONBOY}}$ et al., 2003).

A função da IL-6 na sarcopenia é ainda controversa. Embora estudos epidemiológicos apontem forte associação entre a IL-6 e a perda de massa muscular, trabalhos experimentais não confirmam esta relação (KRABBE et al., 2004). Entretanto, é importante ressaltar que a produção das citocinas IL-6 e TNF- $\alpha$ são intimamente relacionadas, o que talvez apenas permita a observação do efeito produzido por uma citocina em contexto de co-expressão da outra (PETERSEN; PEDERSEN, 2005; FEBBRAIO; PEDERSEN, 2002).

Por outro lado, a adoção do estilo de vida mais ativo e a realização de exercícios físicos parece contribuir com a reversão parcial da sarcopenia. Especula-se que a contração muscular induz a produção e liberação de IL-6 na circulação e contribui com a redução na produção de TNF- $\alpha$ (NICKLAS; BRINKLEY, 2009; STRAUB et al., 2008; FEBBRAIO; PEDERSEN, 2002). Em recente estudo, verificou-se que níveis de TNF foram inversamente relacionados com a força muscular em idosos com fragilidade após 12 semanas de treinamento resistido (BRUUNSGAARD et al., 2004b).

Em conjunto, resultados de estudos sugerem que a atividade física é importante fator para a prevenção e tratamento da sarcopenia. No entanto, é imperativo que novos estudos utilizem desenhos que contemplem tanto a variabilidade polimórfica dos genes de marcadores inflamatórios quanto os hábitos alimentares dos participantes do estudo.

\section{Desempenho cognitivo}

Mecanismos inflamatórios são associados tanto em processos que resultam em declínio cognitivo, incluindo a doença de Alzheimer (DA) e a demência vascular (DV) (HOFMAN et al., 1997), quanto em sintomas de depressão em população geriátrica (PENNINX et al., 2003). Nesta linha, elevados níveis dos mediadores $\mathrm{IL}-6$ e proteína C-reativa (PCR) foram associados a fraco desempenho cognitivo e maior risco de declínio na cognição (YAFFE et al., 2003). Estudos epidemiológicos também descrevem associações entre níveis de TNF- $\alpha$ e DA (TARKOWSKI et al., 2000; TARKOWSKI et al., 2003). Em conjunto, estes resultados sugerem que o sistema nervoso central tanto produz quanto contribui para o pool de citocinas periféricas (KRABBE et al., 2004). Entretanto, é importante ressaltar que elevações centrais nos níveis de citocinas em pacientes com DA e DV não implicam em modelo de causalidade para estes processos patológicos.

Um crescente número de pesquisadores tem investigado a associação de polimorfismos de citocinas com o desenvolvimento de demência, particularmente DA. Porém, os resultados são ainda contraditórios (KRABBE et al., 2004). Por exemplo, resultados de estudos abordando 0 polimorfismo promotor $-174 \mathrm{G} / \mathrm{C}$ do gene IL -6 sugerem tanto reduzida incidência de DA para portadores do alelo C (FALTRACO et al., 2003; POLA et al., 2002) quanto elevada incidência para o referido alelo (LICASTRO et al., 2003). Entretanto, é provável que estas divergências estejam relacionadas com diferenças observadas na distribuição do alelo $C$ entre as populações de pacientes com DA e grupos controle (KRABBE et al., 2004).

Recentemente, foi descrita a associação entre o polimorfismo promotor - $308 \mathrm{G} / \mathrm{A}$ do gene TNF e a demência senil em idosos centenários. $O$ genótipo G/A foi associado à menor prevalência de demência senil quando comparado ao genótipo G/G (BRUUNSGAARD et al., 2004a). Entretanto, resultado de estudo transversal abordando pacientes com DA (60 - 97 anos) e indivíduos de mesma faixa etária e aparentemente saudáveis sugeriu que portadores do alelo apresentam maior risco em relação aos não portadores (ALVAREZ et al., 2002). É provável que esta divergência seja resultado de diferenças na idade dos participantes e/ou no tipo de delineamento adotado.

Apesar da associação entre inflammaging e a redução do desempenho cognitivo, diversos estudos sugerem efeito protetor do exercício frente ao declínio cognitivo. Neste sentido, as revisões de Packer et al. (2010) e Pitkala et al. (2010) ressaltam que o exercício físico é fator de proteção contra a demência, além de auxiliar na reabilitação em indivíduos que possuem disfunção cognitiva. Voss et al. (2010) verificaram que o exercício aeróbio contribui para a maior plasticidade neural em idosos. Já em mulheres pós-menopausa, Eskes et al. (2010) evidenciaram que a preservação das funções cognitivas está diretamente associada ao nível de aptidão física. No entanto, estes benefícios não são observados em adaptações induzidas por exercícios de alta intensidade, uma vez que este proporciona incremento na produção de citocinas próinflamatórias e radicais livres. Cabe destacar que, para intervenções cognitivas de sucesso, a 
variação das modalidades de exercício é mais importante do que a duração da sessão.

Com bases nesses dados, verifica-se a estreita ligação entre processos inflamatórios crônicos relacionados ao envelhecimento e a preservação das funções cognitivas. Entretanto, mais estudos são necessários com o propósito de estabelecer a possível natureza destas relações, bem como de propor estratégias ou intervenções que sejam efetivas para o tratamento ou a reversão deste cenário (KRABBE et al., 2004).

\section{Relação do sistema imunitário e o estado nutricional.}

Quanto aos aspectos nutricionais envolvendo o organismo idoso, sabe-se que tanto a manutenção de um estado nutricional adequado quanto a alimentação equilibrada estão associadas ao envelhecimento saudável. No entanto, muitas são as causas apontadas para explicar as modificações no estado nutricional freqüentemente encontrada neste grupo etário. Idosos podem apresentar alterações sensoriais, digestivas, sociais e afetivas que, em conjunto, produzem graves prejuízos nutricionais com conseqüências ponderais (HARRIS; HABOUBI, 2005). O envelhecimento per se pode vir acompanhado de mudanças na ingestão de alimentos, com conseqüente deficiência de alguns nutrientes ou excesso de outros. Assim surgem quadros de baixo peso e desnutrição ou de sobrepeso e obesidade, frequentes no idoso, com alterações importantes nos parâmetros lipêmicos e glicêmicos (GARIBALLA; SINCLAIR, 1998). Em estudo realizado com diferentes grupos sócioeconômicos, verificou-se que os idosos apresentaram o mesmo padrão alimentar que outros grupos populacionais no tocante aos alimentos energéticos, porém, diferiram quanto aos protéicos e reguladores, os quais ingeriam em menor quantidade (NAJAS et al., 1994).

Pesquisas costumam salientar que a ingestão de carboidratos aumenta com a idade, já que provém de alimentos de baixo custo e mais populares (pães e cereais), no entanto, idosos frequentemente sofrem de problemas mastigatórios (FRANK; SOARES, 2002). Em relação aos micronutrientes, a população idosa na dieta habitual apresenta consumo inadequado dos mesmos, principalmente, de vitamina $\mathrm{A}$ e cálcio (VELASQUES-MELENDEZ et al., 1997).

O cenário exposto pelos trabalhos acima revela grande heterogeneidade na ingestão calórica, o que pode interferir diretamente no perfil imunitário da população. Esta interferência pode decorrer, por exemplo, do fato da gordura visceral produzir e secretar diversas citocinas, que pelo tecido de origem passam a ser denominadas adipocinas. IL-6 e TNF- $\alpha$ são exemplos de adipocinas secretadas ativamente pela massa gorda, em concentrações fisiológicas capazes de modular a resposta imunitária (COPPACK, 2001).

Sendo assim, faz-se necessário que estudos imunogerontológicos tenham a preocupação metodológica de avaliar os sujeitos da pesquisa quanto à sua condição antropométrica (eutrofia/sobrepeso/desnutrição) e composição corporal (relação massa magra/massa gorda) a fim de que a amostra de indivíduos possa ser harmonizada e, por conseqüência, a interferência pelo estado nutricional possa ser dirimida.

\section{Efeitos de prevenção e terapêuticos do exercício físico}

O envelhecimento é descrito como uma complexa remodelagem do organismo, em que alguns parâmetros imunes diminuem enquanto outros permanecem inalterados ou se acentuam. A imunossenescência compreende o fenômeno denominado inflammaging, que é uma regulação positiva da resposta inflamatória por modificação no perfil de citocinas pró-inflamatórias, e que vem sendo relacionado com doenças crônicodegenerativas e com o envelhecimento (GIUNTA, 2006). Em outras palavras, há evidências de que o envelhecimento humano é acompanhado pela elevação dos níveis séricos de diferentes mediadores inflamatórios, o que se postula como contribuinte para as enfermidades consideradas típicas do envelhecimento (hipertensão, diabetes, sarcopenia, fragilidades, etc) (NICKLAS: BRINKLEY, 2009).

Por outro lado, resultados de estudos observacionais evidenciam correlação negativa entre o volume de atividade física e níveis de mediadores inflamatórios circulantes (NICKLAS; BRINKLEY, 2009; STRAUB et al., 2008), ou seja, quanto mais ativo fisicamente o indivíduo menor a chance dos efeitos indesejáveis de citocinas próinflamatórias. Sugere-se que ajustes adaptativos observados em fibras musculares e sistema imunitário inato ao exercício regular contribuem com a redução dos níveis basais de citocinas próinflamatórias, incluindo o TNF- $\alpha$, IFN- $\gamma$ e IL-1 $\beta$ (NICKLAS; BRINKLEY, 2009; PETERSEN; PEDERSEN, 2005). Embora o mecanismo pelo qual o treinamento físico modifica a resposta imunitária seja desconhecido, acredita-se que a liberação aguda de citocinas (miocinas) derivadas de fibras musculares, particularmente IL-6, regule a produção de mediadores inflamatórios liberados por células mononucleares periféricas (NICKLAS; BRINKLEY, 2009) e, indiretamente, por ação no metabolismo de tecidos e órgãos (PEDERSEN; 
FISCHER, 2007). Estudos in vitro têm evidenciado que, durante o processo de envelhecimento monócitos elevam produção de TNF- $\alpha$ (KRABBE et al., 2004) e de citocinas aterogênicas, incluindo INF-Y (NICKLAS; BRINKLEY, 2009). O IFN- $\gamma$ apresenta marcada influência na fisiologia de neutrófilos, por exemplo, elevando sua capacidade fagocítica e microbicida (EDWARDS, 2005). Entretanto, poucos estudos têm investigado a resposta funcional de fagócitos circulantes ao treinamento físico em população idosa.

No âmbito de investigações com populações geriátricas, os resultados são ainda contraditórios (FISCHER, 2006). Por exemplo, BRUUNSGAARD et al. (2004a) observaram que o treinamento resistido melhorou a força muscular, sem, no entanto, alterar significativamente os níveis plasmáticos de TNF- $\alpha$ e IL-6 em idosos com síndrome de fragilidade. Por outro lado, o estudo de Kohut et al. (2006), comparando os efeitos do treinamento aeróbio com o resistido, verificou que, enquanto o primeiro reduziu os níveis de mediadores inflamatórios de forma generalizada, o último provou declínio, exclusivamente, sobre o receptor solúvel do fator de necrose tumoral- $\alpha$ (sTNF- $\alpha$ ). Outro estudo combinando sessões de exercício aeróbio e resistido não reduziu significativamente os níveis de IL- 6 em idosos obesos e mulheres com osteoartrite (NICKLAS et al., 2004). Neste contexto, é provável que divergências observadas sejam, em parte, decorrência de poder insuficiente dos testes power, heterogeneidade genética da amostra, influências nutricionais e ambientais não investigadas (NICKLAS; BRINKLEY, 2009; KRABBE et al., 2004).

\section{Considerações Finais}

Os resultados dos estudos revisados evidenciam progressivos e elevados níveis de citocinas pró-inflamatórias basais que caracterizam o inflammaging. Este processo contribui negativamente para o desencadeamento ou potencialização de doenças crônicas como aterosclerose, sarcopenia, Alzheimer entre outras. Por outro lado, a prática regular de exercícios físicos tem se mostrado eficaz em reduzir os níveis circulantes de mediadores pró-inflamatórios e aumentar a liberação daquelas com atividades antiinflamatórias.

\section{Referências}

ACSM. American College of Sports Medicine Position Stand. Exercise and physical activity for older adults. Medicine and science in sports and exercise, Madison, v. 30, n. 6, p. 992-1008, 2009. Disponível em:
$<$ http://journals.lww.com/acsmmsse/Fulltext/2009/07000/Exercise and Physical Activity for Older Adults.20.aspx>. Acesso em: 11 jul. 2011.

ALVAREZ, A. M.; SCHNEIDER, P.; GONÇALVES, L. H. T. Grupo de ajuda mútua de familiares de idosos portadores de Doença de Alzheimer e doenças similares do HU/UFSC.

Revista Ciências da Saúde, Florianópolis, v. 21, n. 2, p.54-66, 2002.

BAUMGARTNER, R. N.; STAUBER, P .M.; MCHUGH, D.; KOEHLLER, K. M.; GARRY, P. J. Cross-sectional age differences in body composition in persons $60+$ years of age. The journals of gerontology. Series A, Biological sciences and medical sciences, Washington DC, v. 50, n. 6, p. M307-M316, 1995. Disponível em:

$<$ http://biomedgerontology.oxfordjournals.org/cgi/r eprint/50A/6/M307>. Acesso em: 11 jul. 2011.

BELLAVIA, D.; FRADÀ, G.; DI FRANCO, P.; FEO, S.; FRANCESCHI, C.; SANSONI, P.; BRAI, M. C4, BF, C3 allele distribution and complement activity in healthy aged people and centenarians.

The journals of gerontology. Series A, Biological sciences and medical sciences, Washington DC, v.54, n.4, p.B150-153, 1999. Disponível em:

$<$ http://biomedgerontology.oxfordjournals.org/cgi/c ontent/abstract/54/4/B150>. Acesso em: 11 jul. 2011.

BHOJAK, T. J.; DEKOSKY, S. T.; GANGULI, M.; $\mathrm{KAMBOH}, \mathrm{M}$. I. Genetic polymorphisms in the cathespin $D$ and interleukin- 6 genes and the risk of Alzheimer's disease. Neuroscience Letters. Amsterdam, v.288, n.1, p.21-24, 2000. Disponível em:

$<$ http://www.sciencedirect.com/science/article/pii/S 030439400001185X >. Acesso em: 11 jul. 2011.

BONAFÈ, M.; OLIVIERI, F.; CAVALLONE, L.; GIOVAGNETTI, S.; MAYEGIANI, F.; CARDELLI, M.; PIERI, C.; MARRA, M.; ANTONICELLI, R.; LISA, R.; RIZZO, M. R.; PAOLISSO, G.; MONTI, D.; FRANCESCHI, C. Gender-dependent genetic predisposition to produce high levels of IL-6 is detrimental for longevity. European Journal of Immunology, Weinheim, v. 31, n. 8, p. 2357-2361, 2001. Disponível em:

<http://onlinelibrary.wiley.com/doi/10.1002/15214141(200108)31:8\%3C2357::AIDIMMU2357\%3E3.0.CO;2-X/pdf>. Acesso em: 11 jul. 2011.

BRANDT, C.; PEDERSEN, B. K. The role of exercise-induced myokines in muscle homeostasis and the defense against chronic diseases. Journal of biomedicine \& 
biotechnology, Akron, v. 2010, n. 520258, p. 1-6, 2010. Disponível em:

$<$ http://www.ncbi.nlm.nih.gov/pmc/articles/PMC28 36182/pdf/JBB2010-520258.pdf >. Acesso em: 11 jul. 2011.

BRUUNSGAARD, H.; BENFIELD, T.; ANDERSEN-RANBERG, K.; HJELMBORG, J. B.; PEDERSEN, A. N.; SCHROLL, M.; PEDERSEN, B. K.; JEUNE, B. The TNF-308G. The tumor necrosis factor alpha $-308 \mathrm{G}>\mathrm{A}$ polymorphism is associated with dementia in the oldest old. Journal of the American Geriatrics Society, New York, v. 52, n. 8, p. 1361-1366, 2004a. Disponível em: $<$ http://onlinelibrary.wiley.com/doi/10.1111/j.15325415.2004.52369.x/pdf >. Acesso em: 11 jul. 2011.

BRUUNSGAARD, H.; BJERREGAARD, E.; SCHROLL, M.; PEDERSEN, B. K. Muscle strength after resistance training is inversely correlated with baseline levels of soluble tumor necrosis factor receptors in the oldest old. Journal of American Geriatrics Society, New York, v.52, n.2, p.237-241. 2004b. Disponível em: $<$ http://www3.interscience.wiley.com/journal/11874 3865/abstract>. Acesso em 11 jul. 2011.

BRUUNSGAARD, H.; CHRISTIANSEN, L.; PEDERSEN, A. N.; SCHROLL, M.; JORGENSEN, T.; PEDERSEN, B. K. The IL6-174G>C polymorphism is associated with cardiovascular diseases and mortality in 80-year-old humans. Experimental Gerontology, Oxford, v. 39, n. 2, p. 255-261, 2003. Disponível em: $<$ http://www.sciencedirect.com/science/article/pii/S 053155650300281X>. Acesso em: 11 jul. 2011.

BRUUNSGAARD, H.; PEDERSEN, B. K. Agerelated inflammatory cytokines and disease. Immunology and allergy clinics of North America, Philadelphia, v. 23, n. 1, p. 15-39, 2003. Disponível em:

$<$ http://www.immunology.theclinics.com/article/S0 889-8561(02)00056-5/fulltext>. Acesso em: 11 jul. 2011.

BRUUNSGAARD, H.; SKINHOJ, P.; PEDERSEN, A. N.; SCHROLL, M.; PEDERSEN, B. K. Ageing, tumour necrosis factor-alpha (TNF-alpha) and atherosclerosis. Clinical and Experimental Immunology. Oxford, v.121, n.2, p.255-260, 2000. Disponível em:

$<$ http://www.ncbi.nlm.nih.gov/pmc/articles/PMC19 05691/pdf/cei0121-0255.pdf >. Acesso em 11 jul. 2011.

CANDORE, G.; COLONNA-ROMANO, G.; BALESTRIERI, C. R.; DI CARLO, D.; GRIMALDI, M. P.; LISTI, F.; NUZZO, D.; VASTO, S.; LIO, D.; CARUSO, C. Biology of longevity: role of the innate immune system. Rejuvenation Research, Larchmont, v. 9, n. 1, p.143-148, 2006. Disponível em:
$<$ http://www.liebertonline.com/doi/pdf/10.1089/rej. 2006.9.143>. Acesso em: 11 jul. 2011.

CHAVEZ, R. F. A.; HYBKI, P. D. L.; BARBER, R. C.; KEEFE, G. E. Interleukin-6 promoter haplotypes and interleukin- 6 cytokine responses. SHOCK, Augusta, v. 20, n. 3, p. 218-223, 2002. Disponível em:

<http://www.ncbi.nlm.nih.gov/pmc/articles/PMC13 83770/pdf/nihms7296.pdf $>$. Acesso em: 11 jul. 2011.

CONBOY, I. M.; CONBOY, M. J.; SMYTHE, G. M.; RANDO, T. A. Notch-mediated restoration of regenerative potential to aged muscle. Science, New York, v. 302, n. 5650, p.1575-1577, 2003. Disponível em:

$<$ http://seroudelab.biology.queensu.ca/pdf/conboy. pdf>. Acesso em 11 jul. 2011.

COPPACK, S. W. Pro-inflammatory cytokines and adipose tissue. The Proceedings of the Nutrition Society, London, v. 60, n. 3, p. 349356, 2001. Disponível em: $<$ http://journals.cambridge.org/action/displayFullte $x$ t?type $=1 \&$ fid $=804808 \& j i d=\&$ volumeld $=\&$ issueld $=$ 03\&aid $=804796$ \&bodyld $=$ \&membershipNumber $=$ \& societyETOCSession>. Acesso em: 11 jul. 2011.

\section{EDWARDS, S. W. Biochemistry and}

physiology of the neutrophil. New York:

Cambrigde University Press, 2005. Disponível em: $<$ http://www.cambridge.org/catalogue/catalogue.a $\mathrm{sp}$ ?isbn $=9780521018500>$. Acesso em: 11 jul. 2011.

ESKES, G. A.; LONGMAN, S.; BROWN, A. D.; MCMORRIS, C. A.; LANGDON, K. D.; HOGAN, D. B.; POULIN, M. Contribution of physical fitness, cerebrovascular reserve and cognitive stimulation to cognitive function in post-menopausal women. Frontiers in aging neuroscience, Lausanne, $v$. 2, n. 137, p. 1-7, 2010. Disponível em: $<$ http://www.ncbi.nlm.nih.gov/pmc/articles/PMC29 67376/pdf/fnagi-02-00137.pdf >. Acesso em: 11 jul. 2011.

EVANS, W. J. Effects of exercise on body composition and functional capacity of the elderly. The journals of gerontology. Series A, Biological sciences and medical sciences, Washington DC, v. 50, n. special edition, p. 147150, 1995. Disponível em: $<$ http://biomedgerontology.oxfordjournals.org/cgi/r eprint/50A/Special Issue/147>. Acesso em: 11 jul. 2011.

FAGIOLA, U.; COSSARIZZA, A.; SCALA, E.; FANALES-BELASIO, E.; ORTOLANI, C.; COZZI, E.; MONTI, D.; FRANCESCHI, C.; PAGANELLI, $R$. Increased cytokine production in mononuclear cells of healthy elderly people. European Journal of Immunology, Weinheim, v.23, n.9, p.2375- 
2378, 1993. Disponível em:

$<$ http://onlinelibrary.wiley.com/doi/10.1002/eji.1830 230950/abstract>. Acesso em 11 jul. 2011.

FALTRACO, F.; BURGER, K.; ZILL, P.; TEIPEL, S. J.; MOLLER, H. J.; HAMPEL, H.; BONDY, B.; ACKENHEIL, M. Interleukin-6-174 G/C promoter gene polymorphism $\mathrm{C}$ allele reduces Alzheimer's disease risk. Journal of the American Geriatrics Society, New York, v. 51, n. 4, p. 578-579, 2003. Disponível em:

$<$ http://onlinelibrary.wiley.com/doi/10.1046/j.15325415.2003.51177.x/pdf >. Acesso em 11 jul. 2011.

FEBBRAIO, M. A.; PEDERSEN, B. Musclederived interleukin-6: mechanisms for activation and possible biological roles. The FASEB

Journals, Bethesda, v.16, n. 11, p. 1335-1347, 2002. Disponível em:

<http://www.fasebj.org/cgi/reprint/16/11/1335>.

Acesso em: 11 jul. 2011.

FISCHER, C.P. Interleukin-6 in acute exercise and training: what is the biological relevance?

Exercise Immunology Review, Champaign, v. 12, p. 6-33, 2006. Disponível em:

$<$ http://www.medizin.uni-

tuebingen.de/transfusionsmedizin/institut/eir/conte nt/2006/6/article.pdf>. Acesso em: 11 jul. 2011.

FISHMAN, D.; FAULDS, G.; JEFFERY, R.; MOHAMED-ALI, V.; YUDKIN, J. S.; HUMPHRIES, S.; WOO, P. The effect of novel polymorphisms in the interleukin-6 (IL-6) gene on IL-6 transcription and plasma IL-6 levels, and an association with systemic-onset juvenile chronic arthritis. The Journal of Clinical Investigation, New Haven, v.102, n. 7, p.1369-1376, 1998. Disponível em: $<$ http://www.ncbi.nlm.nih.gov/pmc/articles/PMC50 8984/pdf/1021369.pdf>. Acesso em: 11 jul. 2011.

FRANCESCHI, C.; BONAFÈ, M.; VALENSIN, S.; OLIVIERI, F.; de LUCA, M.; OTTAVIANI, E.; De BENEDICTIS, G. Inflammaging. An evolutionary perspective on immunosenescence. Annals of New York Academic Science, New York, v. 9, n. 8, p. 244-254, 2000. Disponível em: $<$ http://onlinelibrary. wiley.com/doi/10.1111/j.17496632.2000.tb06651.x/pdf $>$. Acesso em: 11 jul. 2011.

FRANCESCHI, C.; MONTTI, D.; SANSONI, P.; COSSARIZA, A. The immunology of exceptional individuals: the lesson of centenarians.

Immunology Today, Amsterdam, v. 16, n. 1, p. 12-16, 1995. Disponível em: $<$ http://linkinghub.elsevier.com/retrieve/pii/016756 9995800646>. Acesso em: 11 jul. 2011.

FRANK, A. A.; SOARES, E. A. Nutrição ao envelhecer. São Paulo: Atheneu, 2002. Disponível em:
$<$ http://www.relativa.com.br/livros template.asp?c odigo produto $=9370>$. Acesso em: 11 jul. 2011.

GARIBALLA, S. E.; SINCLAIR, A. J. Nutrition, ageing and ill health. The British Journal of Nutrition, London, v. 80, n. 1, p. 7-23, 1998. Disponível em:

$<$ http://journals.cambridge.org/action/displayFullte xt?type $=1 \&$ fid $=1315236 \& j i d=B J N \&$ volumeld $=80 \& \mathrm{i}$ ssueld=01\&aid=1315228 $>$. Acesso em: 11 jul. 2011.

GAVIN, C.; SIGAL, R. J.; COUSINS, M.; MENARD, M. L.; ATKINSON, M.; KHANDWALA, F.; KENNY, G. P.; PROCTOR, S.; OOI, T. C.; ON BEHALF OF THE DIABETES AEROBIC AND RESISTANCE EXERCISE (DARE) TRIAL INVESTIGATORS. Resistance exercise but not aerobic exercise lowers remnant-like lipoprotein particle cholesterol in type 2 diabetes: A randomized controlled trial. Atherosclerosis, Amsterdam, v. 213, n. 2, p. 552-557, 2010. Disponível em: <http://www.sciencedirect.com/science/article/pii/S 0021915010007197>. Acesso em: 11 jul. 2011.

GEORGES, J. L.; LOUKACI, V.; POIRIER, O.; EVANS, A.; LUC, G.; ARVELIER, D.; RUIDAVETS, J. B.; CAMBIEM, F.; TIRET, L. Interleukin- 6 gene polymorphisms and susceptibility to myocardial infarction the ECTIM study. Journal of Molecular Medicine, Berlin, v. 79, n. 5-6, p. 300-305, 2001. Disponível em: $<$ www.springerlink.com/index/6RC86JG7B5MPLV NA.pdf>. Acesso em: 11 jul. 2011.

GIUNTA, S. Is inflammaging an auto[innate]immunity subclinical syndrome? Immunity \& Ageing, London, v. 3, n. 12, p.1-2, 2006. Disponível em: $<$ http://www.immunityageing.com/content/pdf/1742 -4933-3-12.pdf >. Acesso em: 11 jul. 2011.

HAMERMAN, D. Toward an understanding of frailty. Annals of Internal Medicine, Philadelphia, v.130, n. 11, p. 945-950, 1999. Disponível em: $<$ http://www.annals.org/content/130/11/945.full.pdf $+\underline{h t m l}>$. Acesso em: 11 jul. 2011.

HARRIS, D.; HABOUBI, N. Malnutrition screening in the elderly population. Journal of the Royal Society of Medicine, London, v. 98, n. 9, p. 411414, 2005. Disponível em:

<http://www.ncbi.nlm.nih.gov/pmc/articles/PMC11 99636/pdf/00980411.pdf>. Acesso em: 11 jul. 2011.

HARRIS T. B., FERRUCCI, L.; TRACY, R. P.; CORTI, M. C.; WACHOLDER, S.; ETTINGER, W. $\mathrm{H}$. Associations of elevated interleukin- 6 and creactive protein levels with mortality in the elderly. The American Journal of Medicine, New York, v. 106, n. 5, p. 506-512, 1999. Disponível em: 
$<$ http://www.amimed.com/article/S00029343(99)00066-2/abstract>. Acesso em: 11 jul. 2011.

HOBSON, E. E.; RALSTON, S. H. Role of genetic factors in the pathophysiology and management of osteoporosis. Clinical Endocrinology, Oxford, v. 54, n. 1, p. 1-9. 2001. Disponível em: $<$ http://www3.interscience.wiley.com/cgibin/fulltext/120710496/PDFSTART>. Acesso em: 11 jul. 2011.

HOFMAN, A.; OTT, A.; BRETELER, M. M.; BOTS, M. L.; SLOOTER, A. J.; Van HARSKAMP, F.; Van DUIJIN, C. N.; Van BROECKHOVEN, C.; GROBBEE, D. E. Atherosclerosis, apolipoprotein $\mathrm{E}$, and prevalence of dementia and Alzheimer's disease in the Rotterdam study. Lancet, London, v. 349, n. 9046, p. 151-154, 1997. Disponível em: $<$ http://www.thelancet.com/journals/lancet/article/P IIS0140-6736(96)09328-2/fulltext>. Acesso em: 11 jul. 2011.

HUMPHRIES, S. E.; LUONG, L. A.; OGG, M. S.; HAWE, E.; MILLER, G. J. The interleukin-6 $174 \mathrm{G} / \mathrm{C}$ promoter polymorphism is associated with risk of coronary heart disease and systolic blood pressure in healthy men. European Heart Journal, Oxford, v.22, n.24, p.2243-2252, 2001. Disponível em:

$<$ http://eurheartj.oxfordjournals.org/cgi/reprint/22/2 4/2243.pdf>. Acesso em: 11 jul. 2011.

JENNY, N. S.; TRACY, R. P.; OGG, M. S.; LUONG, L. A.; ARNOLD, A. M.; SHARRET, A. R.; HUMPHRIERS, S. E. In the elderly, interleukin-6 plasma levels and the $-174 \mathrm{G}>\mathrm{C}$ polymorphism are associated with the development of cardiovascular disease. Arteriosclerosis, Thrombosis, and Vascular Biology, Dallas, v. 22, n. 12, p. 2066-2071, 2002. Disponível em: $<$ http://atvb.ahajournals.org/cgi/reprint/22/12/2066 >. Acesso em: 11 jul. 2011.

KOHUT. M. L.; McCANN, D. A.; RUSSELL, D. W.; KONAPKA, D. N.; CUNNICK, J. E.; FRANKE, W. D.; CASTILLO, M. C.; REIGHARD, A. E.; VANDERAH, E. Aerobic exercise, but not flexibility/resistance exercise, reduces serum IL18, CRP, and IL-6 independent of $\beta$-blockers, $\mathrm{BMI}$, and psychological factors in older adults.

Brain, Behavior, and Immunity, San Diego, v. 20, n. 3, p. 201-209, 2006. Disponível em: <http://www.sciencedirect.com/science/article/pii/S 0889159105003727>. Acesso em: 11 jul. 2011.

KRABBE, K. S.; PEDERSEN, M.; BRUUNSGAARD, $\mathrm{H}$. Inflammatory mediators in the elderly. Experimental Gerontology, Oxford, v. 39, n. 5, p. 687-699, 2004. Disponível em: $<$ http://www.sciencedirect.com/science/article/pii/S 0531556504000531>. Acesso em: 11 jul. 2011.
LEUNG, F. P. ; YUNG, L. M.; LAHER, I.; YAO, X.; CHEN, Z. Y.; HUANG, Y. Exercise, vascular wall and cardiovascular diseases: an update (Part 1).

Sports Medicine, Auckland, v. 38, n. 12, p. 10091024, 2008. Disponível em:

$<$ http://adisonline.com/sportsmedicine/pages/articl eviewer. aspx ?year $=2008$ \&issue $=38120$ \&article $=0$ 0005\&type=abstract $>$. Acesso em: 11 jul. 2011.

LICASTRO, F.; LUIGI, M. E. G.; BONAFE, M.; MARTINA, C.; OLIVIERI, F.; CAVALLONE, L.; GIOVANIETTI, S.; MASLIAH, E.; FRANCESCHI, C. Interleukin-6 gene alleles affect the risk of Alzheimer disease and levels of the cytokine in blood and brain. Neurobiology of Aging, New York, v. 24, n. 7, p. 921-926, 2003. Disponível em: $<$ http://www.sciencedirect.com/science/article/pii/S 0197458003000137>. Acesso em: 11 jul. 2011.

LIO, D.; SCOLA, L.; CRIVELLO, A.; COLONNAROMANO, G.; CANDORI G.; BONAFÉ, M.; CAVALLONE, L.; FRAMSESCHI, C.; CARUSO, C.; Gender-specific association between -1082 IL10 promoter polymorphism and longevity. Genes and Immunity, Houndmills, v. 3, n. 1, p.30-33, 2002. Disponível em: $<$ http://www.nature.com/gene/journal/v3/n1/pdf/63 63827a.pdf >. Acesso em: 11 jul. 2011.

MARRIOTTI, S.; FRANCESCHI, C.; COSSARIZZA, A.; PINCHERA, A. The aging thyroid. Endocrine Reviews, Baltimore, v. 16, n. 6, p. 686-715, 1995. Disponível em: $<$ http://edrv.endojournals.org/content/16/6/686.abs tract>. Acesso em: 11 jul. 2011.

MIERS, J. Cardiology patient pages. Exercise and cardiovascular health. Circulation, Dallas, v. 107, n. 1, e2-e5, 2003. Disponível em: <http://circ.ahajournals.org/cgi/reprint/107/1/e2>. Acesso em: 11 jul. 2011.

NAJAS, M.S.; ANDREAZZA, R.; SOUSA, A.L.M.; SACHS, A.; GUEDES, A.C.B.; SAMPAIO, L.R. Padrão alimentar de idosos de diferentes estratos socioeconômicos residentes em localidade urbana da região sudoeste. Revista de Saúde Pública, São Paulo, n. 28, v. 3, p.187-91, 1994. Disponível em: $<$ http://www.scielo.br/pdf/rsp/v28n3/04.pdf>. Acesso em: 11 jul. 2011.

NICKLAS, B. J.; AMBROSIUS, W.; MESSIER, S. P.; MILLER, G. D.; PENNINX, B. W. J. H.; LOESER, R. F.; PALLA, S.; BLEECKER, E.; PAHOR, M. Diet-induced weight loss, exercise, and chronic inflammation in older, obese adults: a randomized controlled clinical Trial. The American Journal Clinical of Nutrition, Bethesda, v.79, n.4, p.544-551, 2004. Disponível em: <http://www.ajcn.org/cgi/reprint/79/4/544>. Acesso em: 11 jul. 2011. 
NICKLAS, B.J.; BRINKLEY, T.J. Exercise Training as a Treatment for Chronic Inflammation in the Elderly. Exercise and Sports Science Review, New York, v.37, n.4, p.165-170, 2009. Disponível em: <http://journals.lww.com/acsmessr/Abstract/2009/10000/Exercise Training as a Treatment for Chronic.4.aspx>. Acesso em: 11 jul. 2011.

PACKER N.; PERVAIZ, N.; HOFFMAN-GOETZ, L. Does exercise protect from cognitive decline by altering brain cytokine and apoptotic protein levels? A systematic review of the literature. Exercise Immunology Review, Champaign, v. 16, p.138-162, 2010. Disponível em:

$<$ http://www.medizin.unituebingen.de/transfusionsmedizin/institut/eir/conte nt/2010/138/article.pdf >. Acesso em: 11 jul. 2011.

PEDERSEN, B. K.; FISCHER, C. P. Beneficial health effects of exercise - the role of IL-6 as a myokine. Trends in Pharmacological Sciences, Amsterdam, v. 28, n. 4, p. 152-56, 2007.

Disponível em: <http://inflammationmetabolism.dk/misc/download.php?url=../files/repr ints/PMID\%2017331593.pdf\&type=txt>. Acesso em: 11 jul. 2011.

PENNINX, B. W.; KRITCHEVSKY, S. B.; YAFFE, K.; NEWMAN, A. B.; SIMONSICK, E. M.; RUBIN, S.; FERRUCCI, L.; HARRIS, T.; PAHOR, M. Inflammatory markers and depressed mood in older persons: results from the health, aging and body composition study. Biological Psychiatry, New York, v. 54, n. 5, p. 566-572, 2003.

Disponível em:

$<$ http://www.journals.elsevierhealth.com/periodical s/bps/article/S0006-3223(02)01811-5/pdf >.

Acesso em: 11 jul. 2011.

PEPYS, M. B.; HIRSCHFIELD, G. M. C-reactive protein: a critical update. The Journal of Clinical Investigation, New Haven, v. 111, n. 12, p. 18051812, 2003. Disponível em:

$<$ http://www.jci.org/articles/view/18921/pdf>.

Acesso em: 11 jul. 2011.

PETERSEN, A. M. W.; PEDERSEN, B. K. The anti-inflammatory effect of exercise. Journal of applied Physiology, Bethesda, v. 98, n. 4, p. 1154-1162, 2005. Disponível em: $<$ http://jap.physiology.org/cgi/reprint/98/4/1154>. Acesso em 11 jul. 2011.

PITKALA, K. H.; RAIVIO, M. M.; LAAKKONEN, M. L.; TILVIS, R. S.; KAUTIAINEN, H.;

STRANDBERG, T. E. Exercise rehabilitation on home-dwelling patients with Alzheimer's disease a randomized, controlled trial. Study protocol.

Trials, London, v. 11, n. 92, p. 1-7, 2010.

Disponível em:

$<$ http://www.ncbi.nlm.nih.gov/pmc/articles/PMC29 78202/pdf/1745-6215-11-92.pdf >. Acesso em: 11 jul. 2011.
POLA, R.; FLEX, A.; GAETANI, E.; LAGO, A. D.; GERARDINO, L.; POLA, P.; BERNABEI, R. The $174 \mathrm{G} / \mathrm{C}$ polymorphism of the interleukin- 6 gene promoter is associated with Alzheimer's disease in an Italian population. Neuroreport, Oxford, v. 13, n. 13, p.1645-1647, 2002. Disponível em: $<$ http://journals.Iww.com/neuroreport/pages/article viewer. asp $x$ ?year $=2002 \&$ issue $=09160$ \&article $=00$ 015\&type=abstract $>$. Acesso em: 11 jul. 2011.

PRAVICA, V.; ASDERAKIS, A.; PERREY, C.; HAJEER, A.; SINNOTT, P. J.; HUTCHINSON, I. $V$. In vitro production of IFN-gamma correlates with CA repeat polymorphism in the human IFNgamma gene. European Journal of Immunogenetics, Oxford, v. 26, n. 1, p. 1-3, 1999. Disponível em:

$<$ http://onlinelibrary.wiley.com/doi/10.1046/j.13652370.1999.00122.x/pdf>. Acesso em : 11 jul. 2011.

ROTH, S. M.; FERREL, R. E.; HURLEY, B. F. Strength training for the prevention and treatment of sarcopenia. Jornal of Nutrional, Health and Aging, Paris, v. 4, n. 3, p. 143-155, 2000. Disponível em: $<$ http://kioa.keiser.com/research/Abstracts/roth st sarcopenia.pdf>. Acesso em: 11 jul. 2011.

ROTH, S. M.; METTER, E. J.; LING, S.;

FERRUCCI, L. Inflammatory factors in age-related muscle wasting. Current Opinion in

Rheumatology, Philadelphia, v. 18, n. 6, p. 625630, 2006. Disponível em:

$<$ http://journals.Iww.com/co-

rheumatology/Abstract/2006/11000/Inflammatory factors in age related muscle wasting.11.aspx> . Acesso em: 11 jul. 2011.

ROUBENOFF, R. Catabolism of aging: is it an inflammatory process? Current Opinion in Clinical Nutrition and Metabolic Care, London, v. 6, n. 3, p. 295-299, 2003. Disponível em: $<$ http://journals.Iww.com/coclinicalnutrition/Abstract/2003/05000/Catabolism of aging is it an inflammatory.5.aspx>. Acesso em: 11 jul. 2011.

ROUBENOFF, R.; HUGUES, V. A. Sarcopenia: current concepts. The journals of gerontology. Series A, Biological sciences and medical sciences. Washington DC, v. 55, n. 12, M716724, 2000. Disponível em:

$<$ http://biomedgerontology.oxfordjournals.org/cgi/r eprint/55/12/M716>. Acesso em: 11 jul. 2011.

SANSONI, P.; COSSARIZZA, A.; BRIANTI, V.; FAGNONI, F.; SNELLI, G.; MONTI, D.; MARCATO, A.; PASSERI, G.; ORTOLANI, C.; FORTI, E. Lymphocyte subsets and natural killer cell activity in healthy old people and centenarians. Blood, Washington DC, v. 82, n. 9, p. 2767-2773, 1993. Disponível em: 
$<$ http://bloodjournal.hematologylibrary.org/cgi/repri nt/82/9/2767>. Acesso em: 11 jul. 2011.

SKOOG, T.; DICHTL, W.; BOQUIST, S.; SKOGLUND-ANDERSON, C.; KARPE, F.; TANG, R.; BOND, M. G.; FAIRE, U.; NILSON, J.; ERIKSSON, P.; HAMSTEN, A. Plasma tumour necrosis factor-alpha and early carotid atherosclerosis in health middle-aged men.

European Heart Journal, Oxford, v. 23, n. 5, p. 376-383, 2002. Disponível em: $<$ http://eurhearti.oxfordjournals.org/cgi/reprint/23/5 /376.pdf >. Acesso em: 11 jul. 2011.

STRAUB, R. H.; TANKÓ, L. B.; CHRISTIANSEN, C.; LARSEN, P. J.; JESSOP, D.S. Higher physical activity is associated with increased androgens, low interleukin 6 and less aortic calcification in peripheral obese elderly women. The Journal of Endocrinology, London, v. 199, n. 1, p. 61-68, 2008. Disponível em: $<$ http://joe.endocrinologyjournals.org/content/199/1/61.full.pdf+html >. Acesso em: 11 jul. 2011.

TARKOWSKI, E.; LILJEOTH, A. M.; MINTHON, L.; TARKOWSKI, A.; WALLIN, A.; BLENNOW, K. Cerebral pattern of pro- and anti-inflammatory cytokines in dementias. Brain Research Bulletin, New York, v. 61, n. 3, p. 255-260, 2003.

Disponível em:

$<$ http://www.sciencedirect.com/science/article/pii/S 0361923003000881>. Acesso em: 11 jul. 2011.

TARKOWSKI, E.; LILJEOTH, A.M.; NILSSON, A.; RICKSTEN, A.; DAVIDSSON, P.; MINTHON, L.; BLENNOW, K. TNF gene polymorphism and its relation to intracerebral production of TNF alpha and TNF beta in AD. Neurology. Minneapolis, v.54, n.11, p.2077-2081, 2000. Disponível em: $<$ http://www.neurology.org/content/54/11/2077.abs tract?sid=246fc5bc-3cde-45ad-879d-

25aea8385adb >. Acesso em: 11 jul.2011.

TERRY, C. F.; LOUKACI, V.; GREEN, F. R. Cooperative influence of genetic polymorphisms on interleukin- 6 trascriptional regulation. The Journal Biological Chemistry. Baltimore, v. 275, n. 24, p. 1838-1844, 2000. Disponível em:<http://www.jbc.org/content/275/24/18138.full. pdf $>$. Acesso em: 11 jul. 2011.

VELASQUES-MELENDEZ, G.; SALAS MARTINS, I.; CERVATOA, M. Consumo alimentar de vitaminas e minerais em adultos residentes em área metropolitana de São Paulo, Brasil. Revista de Saúde Pública, São Paulo, n. 31, v. 2, p. 157162, 1997. Disponível em: <http://www.scielo.br/pdf/rsp/v31n2/2223.pdf>. Acesso em: 11 jul. 2011.

VOSS, M. W.; PRAKASH, R. S.; ERICKSON, K. I.; BASAK, C.; CHADDOCK, L.; KIM, J. S.;

ALVES, H.; HEO, S.; SZABO, A. N.; WHITE, S.
M.; WÓJCICKI, T. R.; MAILEY, E. L.; GOTHE, N.; OLSON, E. A.; MCAULEY, E.; KRAMER, A. F. Plasticity of brain networks in a randomized intervention trial of exercise training in older adults. Frontiers in Aging Neuroscience, Lausanne, v. 2, n. 32, p.1-17, 2010. Disponível em:

$<$ http://www.ncbi.nlm.nih.gov/pmc/articles/PMC29 47936/pdf/fnagi-02-00032.pdf >. Acesso em: 11 jul. 2011.

YAFFE, K.; LINDQUIST, K.; PENNINX, B. W.; SIMONSICK, E. M.; PAHOR, M.; KRICHEVSKY, S.; LAUNER, L.; KULLER, L.; RUBIN, S.; HARRIS, T. Inflammatory markers and cognition in well-functioning African-American and white elders. Neurology, Minneapolis, v. 61, n. 1, p. 7680, 2003. Disponível em:

$<$ http://www.neurology.org/content/61/1/76.abstrac $\mathrm{t}$ ?sid=462b31d1-2e34-45c5-8416-

65621831e000>. Acesso em: 11 jul. 2011.

ZHANG, M. H.; NA, B.; SCHILLER, N. B.; WHOOLEY, M. A. Resistin, exercise capacity, and inducible ischemia in patients with stable coronary heart disease: Data from the Heart and Soul

study. Atherosclerosis, Amsterdam. v. 213, n. 2, p. 604-610, 2010. Disponível em:

$<$ http://www.sciencedirect.com/science/article/pii/S 0021915010007434 >. Acesso em: 11 jul.2011.

Endereço:

Ciro José Brito

Rua São Felipe 32/202 - Sagrada Família

Belo Horizonte, MG, Brasil

31030290.

e-mail: cirojbrito@gmail.com

Recebido em: 23 de fevereiro de 2010.

Aceito em: 12 de maio de 2011.

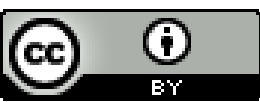

Motriz. Revista de Educação Física. UNESP, Rio Claro, SP, Brasil - elSSN: 1980-6574 - está licenciada sob Creative Commons - Atribuição 3.0 